\title{
How Many Users Are Needed for Non-Trivial Performance of Random Beamforming in Highly-Directional mm-Wave MIMO Downlink?
}

\author{
Gilwon Lee, Youngchul Sung ${ }^{\dagger}$, and Junyeong Seo \\ Dept. of Electrical Engineering \\ KAIST \\ Daejeon, South Korea 305-701 \\ E-mail:\{gwlee@,ysung@ee., and jyseo@\}kaist.ac.kr.
}

\begin{abstract}
In this paper, the multi-user (MU) gain and the impact of training are investigated for random beamforming (RBF) in highly-directional millimeter-wave (mm-wave) MU multipleinput multiple-output (MIMO) downlink which is considered as one of the major technological thrusts for $5 \mathrm{G}$ communications. To do so, the uniform random line-of-sight (UR-LoS) channel model suitable for highly-directional mm-wave radio propagation channels is adopted. Based on the UR-LOS channel model, the performance of $R B F$ and the MU gain in mm-wave MIMO systems are analyzed. It is shown that there exists a transition point on the number of users relative to the number of transmit antennas for non-trivial performance of the RBF scheme. Furthermore, in case of training, the number of training beams relative to the number of antennas required for asymptotically good performance of the RBF scheme is specified when the number of users relative to the number of antennas is given. The provided results in this paper yields insights into the MU gain with partial CSI and the amount of required training in highly-directional mm-wave channels.
\end{abstract}

\section{INTRODUCTION}

Random beamforming with partial CSI was proposed for MU-MIMO downlink to circumvent the problem of channel estimation and feedback of channel state information (CSI) in [3]. In this scheme, the transmitter picks a random beam or a set of random orthogonal beams for data transmission and exploits the MU gain in the network to yield reasonable performance based only on partial CSI. Due to such advantages, random beamforming and the associated MU gain have been investigated extensively during the last decade [3][7]. However, most of analysis was performed under rich scattering environments which are characteristics of low radio frequency bands. Recently, mm-wave MIMO operating in the band of $30-300 \mathrm{GHz}$ is rising as a candidate technology for $5 \mathrm{G}$ wireless communications to meet exponentially-growing wireless capacity demands, and there are several challenging issues to realize mm-wave MIMO systems. One of such issues is to estimate mm-wave MIMO CSI [8]-[10]. The radio propagation in the mm-wave band has quasi-optical nature with large path loss and very few multi-paths, and the MIMO channel in the mm-wave band is sparse in the arrival angle domain [8], [9]. Thus, it is difficult to identify the CSI between the transmitter and an arbitrary user in the network with a

This work was supported by ICT R\&D program of MSIP/IITP. [B0101-151309, Development of Adaptive Beam Multiple Access Technology without Interference based on Antenna Node Grouping]. The journal version of this paper is available at http://arxiv.org/abs/1412.1665 [1] and a related paper is in Proc. of IEEE SPAWC 2015 [2]. highly-directional training beam compensating for the large path loss in the mm-wave band. Identifying CSI requires sophisticated algorithms and heavy training overhead [8]-[10], and feedback of the identified CSI is another issue.

In this paper, to circumvent such difficulties, we consider random beamforming with partial CSI for mm-wave MUMIMO downlink and investigate its performance and associated MU gain in the mm-wave MIMO environment with highly-directional quasi-optical propagation characteristics. To analyze the performance of random beamforming in mm-wave MU-MIMO downlink, we adopt the uniform random line-ofsight (UR-LoS) channel model which well captures the highlydirectional propagation characteristics in the mm-wave band [11], [12]. Under this channel model, we reveal a transition behavior of the random beamforming performance and the interplay between the number of users and the amount of training for random beamforming in higly-directional $\mathrm{mm}$ wave MU-MIMO downlink. The results in this paper provide insights into the MU gain with partial CSI in highly-directional mm-wave MU-MIMO downlink systems.

Notations and Organization: In this paper, we will use standard notational conventions. Vectors and matrices are written in boldface with matrices in capitals. For a matrix $\mathbf{A}, \mathbf{A}^{T}$, $\mathbf{A}^{H}$, and $\operatorname{tr}(\mathbf{A})$ indicate the transpose, conjugate transpose, and trace of $\mathbf{A}$, respectively. The notation $x \sim \mathcal{C N}(0,1)$ means that $x$ is the complex Gaussian random variable with mean 0 and variance 1 , and $\theta \sim \operatorname{Unif}[a, b]$ means that $\theta$ is uniformly distributed over the range $[a, b] . \mathbb{E}[\cdot]$ denotes the expectation. $\iota:=\sqrt{-1}$ and $\mathbb{Z}$ is the set of integers.

\section{System Model}

We consider a single-cell mm-wave MU-MIMO downlink system where a transmitter equipped with a uniform linear array (ULA) of $M$ transmit antennas communicates with $K$ single-antenna users. The received signal at user $k$ is then given by

$$
y_{k}=\mathbf{g}_{k}^{H} \mathbf{x}+n_{k}, \quad k=1,2, \cdots, K,
$$

where $\mathbf{g}_{k}$ is the channel vector of user $k, \mathbf{x}$ is the transmitted signal vector subject to a power constraint $\operatorname{tr}\left(\mathbb{E}\left\{\mathbf{x x}^{H}\right\}\right) \leq P_{t}$, and $n_{k} \sim \mathcal{C N}(0,1)$ is the additive noise at user $k$.

Due to the highly directional and quasi-optical nature of electromagnetic wave propagation in the mm-wave band, a 
typical mm-wave channel has very few multi-path components. In general, a mm-wave channel is composed of a lineof-sight (LoS) propagation component and possibly a set of few single-bounce non-LoS (NLoS) components [13]. Hence, the mm-wave channel of user $k$ can be modeled as [11]

$$
\mathbf{g}_{k}=\alpha_{k} \sqrt{M} \mathbf{a}\left(\theta_{k}\right)+\sum_{i} \alpha_{k, i} \sqrt{M} \mathbf{a}\left(\theta_{k, i}\right)
$$

where $\alpha_{k}$ and $\theta_{k}$ are the complex gain and normalized direction of the LoS path for user $k,\left\{\alpha_{k, i}\right\}$ and $\left\{\theta_{k, i}\right\}$ represent the complex gains and normalized directions of NLoS paths for user $k$, and $\mathbf{a}(\theta)$ is the array steering vector given by

$$
\mathbf{a}(\theta)=\frac{1}{\sqrt{M}}\left[1, e^{-\iota \pi \theta}, \cdots, e^{-\iota \pi(M-1) \theta}\right]^{T} .
$$

Here, the normalized direction $\theta$ is related to the physical angle of departure $\phi \in[-\pi / 2, \pi / 2]$ as $\theta=\frac{2 d \sin (\phi)}{\lambda}$, where $d$ and $\lambda$ are the distance between two adjacent antennas and the carrier wavelength, respectively. We simply assume $\frac{d}{\lambda}=\frac{1}{2}$. Note that the array steering vector in (3) has unit norm and hence the normalization factor $\sqrt{M}$ is included in (2).

Since the path loss of NLoS components is much larger than that of the LoS component, i.e., the power $\left|\alpha_{k, i}\right|^{2}$ associated with NLoS paths is typically $20 \mathrm{~dB}$ weaker than the LoS component $\left|\alpha_{k}\right|^{2}$ [13], the effect of NLoS links is negligible for mm-wave channels with LoS links. Hence, we focus on LoS channels here, i.e., $\alpha_{k, i}=0$ for $\forall i$ [11], [14]. Furthermore, we assume that the gain associated with the LoS link is Gaussian-distributed, i.e., $\alpha_{k} \stackrel{\text { i.i.d. }}{\sim} \mathcal{C N}(0,1)$ and that the normalized direction $\theta_{k}$ for each user $k$ is i.i.d. with $\theta_{k} \stackrel{i . i . d .}{\sim}$ Unif $[-1,1]$. From the above assumptions, the mmwave channel model (2) can be re-written as

$$
\mathbf{g}_{k}=\alpha_{k} \sqrt{M} \mathbf{a}\left(\theta_{k}\right),
$$

for $k=1, \cdots, K$. This channel model is the uniform random line-of-sight (UR-LoS) model already considered for mmwave MIMO in [11], [12]. In this paper, we adopt the UR-LoS model to make analysis tractable and gain insights into RBF in highly-directional MIMO channel environments.

\section{RANDOMLY-DIRECTIONAL BEAMFORMING IN MM-WAVE MIMO}

In highly-directional mm-wave MIMO, the randomness in random beamforming lies in direction. Thus, we first consider the following randomly-directional beamforming (RDB) strategy in downlink transmission.

Step 1) During the training period, the transmitter chooses a normalized direction $\vartheta$ randomly and broadcasts the beam $\mathbf{x}$ in (1) given by

$$
\mathbf{x}=\mathbf{a}(\vartheta)
$$

where $\vartheta \sim \operatorname{Unif}[-1,1]$. (We set $P_{t}=1$ for simplicity here.)

Step 2) Each user $k$ in the cell computes and feedbacks the average received power* $\left|\bar{y}_{k}\right|^{2}\left(\approx\left|\mathbf{g}_{k}^{H} \mathbf{x}\right|^{2}+\frac{1}{N_{s}}\right)$ to the transmitter, where $\left|\mathbf{g}_{k}^{H} \mathbf{x}\right|^{2}=\left|\alpha_{k}\right|^{2} \cdot M\left|\mathbf{a}\left(\theta_{k}\right)^{H} \mathbf{a}(\vartheta)\right|^{2}$.

\footnotetext{
* To average out the noise effect, each user can exploit multiple time samples during the training period for the feedback value $\left|\bar{y}_{k}\right|^{2}$, where $\bar{y}_{k}$ is the sample average.
}

Step 3) After the feedback period, the transmitter selects the user that has the maximum received signal power and transmits a data stream with the beamforming vector $\mathbf{x}$ in (5) to the user.

The considered random beamforming requires partial CSI, i.e., not the full CSI $\mathbf{g}_{k}$ but a real number capturing the channel's alignment to a given random transmit beam from each user. The expected rate $\mathcal{R}_{1}$ of the RDB scheme is given by

$$
\mathcal{R}_{1}=\mathbb{E}\left[\log \left(1+\max _{1 \leq k \leq K}\left|\alpha_{k}\right|^{2} M\left|\mathbf{a}\left(\theta_{k}\right)^{H} \mathbf{a}(\vartheta)\right|^{2}\right)\right] .
$$

To gain insights, first consider the RDB rate in the case of $K=1$. In this case, we have an upper bound on $\mathcal{R}_{1}$ from Jensen's inequality, given by

$$
\begin{aligned}
\mathcal{R}_{1} & =\mathbb{E}\left[\log \left(1+\left|\alpha_{1}\right|^{2} M\left|\mathbf{a}\left(\theta_{1}\right)^{H} \mathbf{a}(\vartheta)\right|^{2}\right)\right] \\
& \leq \log \left(1+\mathbb{E}\left[\left|\alpha_{1}\right|^{2} M\left|\mathbf{a}\left(\theta_{1}\right)^{H} \mathbf{a}(\vartheta)\right|^{2}\right]\right)=\log 2 .
\end{aligned}
$$

The last equality holds by $\mathbb{E}\left[\left|\alpha_{1}\right|^{2}\right]=1$ since $\left|\alpha_{1}\right|^{2}$ has a chisquare distribution with degree-of-freedom two, i.e., $\left|\alpha_{1}\right|^{2} \sim$ $\chi^{2}(2)$ and

$$
\begin{aligned}
\mathbb{E}\left[M\left|\mathbf{a}\left(\theta_{1}\right)^{H} \mathbf{a}(\vartheta)\right|^{2}\right] & =\frac{1}{M} \mathbb{E}\left[\left|\sum_{n=0}^{M-1} e^{-\iota \pi n\left(\vartheta-\theta_{1}\right)}\right|^{2}\right] \\
& =\frac{1}{M} \mathbb{E}\left[M+\sum_{\substack{n, m \\
n \neq m}} e^{-\iota \pi(m-n)\left(\vartheta-\theta_{1}\right)}\right] \\
& =1 .
\end{aligned}
$$

The last step is valid since $\mathbb{E}\left[e^{-\iota \pi(m-n)\left(\vartheta-\theta_{1}\right)}\right]=$ $\frac{1}{2} \int_{-1}^{1} e^{-\iota \pi(m-n) \tilde{\theta}_{k}} d \tilde{\theta}_{k}=\frac{\sin \pi(m-n)}{\pi(m-n)}=0$ for any $(m-n) \in$ $\mathbb{Z} \backslash\{0\}[12] .{ }^{\dagger}$ Hence, the rate of the RDB scheme for $K=1$ is insignificant irrespective of the value of $M$. In this case, one should acquire the CSI of the single user to achieve the rate with perfect CSI given by

$$
\log \left(1+\left|\alpha_{1}\right|^{2} M\right) \sim \log M,
$$

and the channel estimation is important [8]-[10]. (In this paper, $x \sim y$ indicates $\lim _{M \rightarrow \infty} x / y=1$.)

\section{Asymptotic Analysis of The RDB Rate}

In the previous section, we have seen that the performance of the RDB scheme is trivial when $K=1$. Now consider the case where $K$ becomes large. Consider the term $\left|\mathbf{a}\left(\theta_{k}\right)^{H} \mathbf{a}(\vartheta)\right|$ in the right-hand side (RHS) of (6):

$$
\begin{aligned}
\left|\mathbf{a}\left(\theta_{k}\right)^{H} \mathbf{a}(\vartheta)\right| & =\frac{1}{M}\left|\sum_{n=0}^{M-1} e^{-\iota \pi n\left(\vartheta-\theta_{k}\right)}\right|=\frac{1}{M}\left|\frac{1-e^{-\iota \pi\left(\vartheta-\theta_{k}\right) M}}{1-e^{-\iota \pi\left(\vartheta-\theta_{k}\right)}}\right| \\
& =\frac{1}{M}\left|\frac{\sin \frac{\pi\left(\vartheta-\theta_{k}\right) M}{2}}{\sin \frac{\pi\left(\vartheta-\theta_{k}\right)}{2}}\right|=: F_{M}\left(\vartheta-\theta_{k}\right),
\end{aligned}
$$

which is called the Fejér kernel $F_{M}(\cdot)$ of order $M$ [15]. From (9), for fixed $\vartheta$ and $\theta_{k}$ we have $\left|\mathbf{a}\left(\theta_{k}\right)^{H} \mathbf{a}(\vartheta)\right| \rightarrow 0$ and thus $\mathcal{R}_{1} \rightarrow 0$ as $M \rightarrow \infty$. However, if we can find user $k$ such that

\footnotetext{
${ }^{\dagger}$ We can regard $\tilde{\theta}_{k}:=\vartheta-\theta_{k} \sim \operatorname{Unif}[-1,1]$ in case that $\vartheta-\theta_{k}$ appears as $e^{\iota \pi l\left(\vartheta-\theta_{k}\right)}$ for any integer $l$ due to the periodicity of period two.
} 
$\vartheta-\theta_{k}=\frac{\Delta}{M}$ for some $\Delta>0$, then $\left|\mathbf{a}\left(\theta_{k}\right)^{H} \mathbf{a}(\vartheta)\right| \rightarrow \mid \frac{2 \sin \frac{\pi \Delta}{2}}{\pi \Delta}$ as $M \rightarrow \infty$ and a nontrivial rate is achievable by the RDB scheme [12].

In this section, we analyze the asymptotic performance of the RDB scheme in a massive MIMO asymptote, i.e., $M \rightarrow \infty$. It is difficult to directly compute $\mathcal{R}_{1}$ in (6) because several random variables in the integral in (6) are intertwined. To circumvent this difficulty, we apply asymptotic techniques to bound $\mathcal{R}_{1}$ by first assuming that $\alpha_{k}=1$ for all $k$ and focusing on the term $Z_{k}:=M\left|\mathbf{a}\left(\theta_{k}\right)^{H} \mathbf{a}(\vartheta)\right|^{2}$ in (6). Then, we will take into account the term $\alpha_{k} \sim \mathcal{C N}(0,1)$ in the analysis later. We first provide the key lemma in the following.

Lemma 1: [1] For any constant $p \in(-1,1)$ and sufficiently large $M$, we have

$$
\frac{1}{2 \pi M^{(1+p) / 2}}<\operatorname{Pr}\left\{Z_{k}>M^{p}\right\}<\frac{1}{\frac{\pi}{4} M^{(1+p) / 2}} .
$$

That is, $\operatorname{Pr}\left\{Z_{k}>M^{p}\right\}=\Theta\left(\frac{1}{M^{(1+p) / 2}}\right)$.

Proof: From (9), the following two events are equivalent:

$$
\begin{aligned}
& Z_{k}=M\left|\mathbf{a}\left(\theta_{k}\right)^{H} \mathbf{a}(\theta)\right|^{2}>M^{p} \\
\Leftrightarrow & \left|\frac{\sin \frac{\pi \tilde{\theta}_{k} M}{2}}{\sin \frac{\pi \tilde{\theta}_{k}}{2}}\right|>M^{(1+p) / 2},
\end{aligned}
$$

where $\tilde{\theta}_{k} \sim \operatorname{Unif}[-1,1]$. Since the numerator $\left|\sin \frac{\pi \tilde{\theta}_{k} M}{2}\right| \leq 1$ and $M^{(1+p) / 2}>1$ for $p \in(-1,1)$, a necessary condition to satisfy (11) is that the denominator in the left-hand side (LHS) of (11) should be upper bounded as

$$
\left|\sin \frac{\pi \tilde{\theta}_{k}}{2}\right|<\frac{1}{M^{(1+p) / 2}} .
$$

For given $p \in(-1,1)$, the upper bound in the RHS of (12) tends to zero as $M \rightarrow \infty$. Therefore, by the fact that $\frac{\epsilon}{2}<\sin \epsilon$ for small $\epsilon>0$, (12) implies

$$
\left|\tilde{\theta}_{k}\right|<\frac{1}{\frac{\pi}{4} M^{(1+p) / 2}}
$$

for sufficiently large $M$. Therefore, we have

$$
\operatorname{Pr}\left\{Z_{k}>M^{p}\right\}<\operatorname{Pr}\left\{\left|\tilde{\theta}_{k}\right|<\frac{1}{\frac{\pi}{4} M^{(1+p) / 2}}\right\}=\frac{1}{\frac{\pi}{4} M^{(1+p) / 2}},
$$

since $\tilde{\theta}_{k} \sim \operatorname{Unif}[-1,1]$.

Now we consider the lower bound in (10). From the fact that $\sin \epsilon<\epsilon$ for $\epsilon>0$, we have

$$
\left|\sin \frac{\pi \tilde{\theta}_{k}}{2}\right|<\frac{1}{2 M^{(1+p) / 2}}
$$

if

$$
\frac{\pi}{2}\left|\tilde{\theta}_{k}\right|<\frac{1}{2 M^{(1+p) / 2}} .
$$

If the following equation holds in addition to (15) (implying (14))

$$
\left|\sin \frac{\pi \tilde{\theta}_{k} M}{2}\right| \geq \frac{1}{2}
$$

then (11) is satisfied. But, (16) is satisfied when

$$
\left|\tilde{\theta}_{k}\right| \in\left\{\left[\frac{1}{3 M}+\frac{2 k}{M}, \frac{5}{3 M}+\frac{2 k}{M}\right], k=0,1,2, \cdots,\right\} .
$$

Note that $\left|\sin \frac{\pi \tilde{\theta}_{k} M}{2}\right|$ in (16) has period $\frac{2}{M}$ and the length of one interval per period in the set (17) is $\frac{4}{3 M}$. Hence, the set (17) occupies $\frac{2}{3}$ length of each period of $\frac{2}{M}$. Since the term $\frac{1}{M^{(p+1) / 2}}$ for given $p \in(-1,1)$ converges to zero slower than $\frac{1}{M}$ as $M \rightarrow \infty$, multiple discontinuous intervals in the set (17) are contained in the set defined by (15), and the length of the intersection of the sets (15) and (17) is lower bounded by $\frac{2}{3}\left(\frac{1}{\pi M^{(1+p) / 2}}-\frac{2}{M}\right)$, where minus $\frac{2}{M}$ takes into account the impact of the last possibly partially overlapping interval. Therefore, we have

$$
\begin{aligned}
\operatorname{Pr}\left\{Z_{k}>M^{p}\right\} & \geq \frac{2}{3}\left(\frac{1}{\pi M^{(1+p) / 2}}-\frac{2}{M}\right) \\
& >\frac{1}{2} \cdot \frac{1}{\pi M^{(1+p) / 2}},
\end{aligned}
$$

for $M$ is sufficiently large. This concludes the proof.

Based on Lemma 1 we now have the following theorem regarding the asymptotic performance of the RDB scheme.

Theorem 1: [1] For $K=M^{q}$ and $q \in(0,1)$, we have asymptotic upper and lower bounds for $\mathcal{R}_{1}$ in (6) in the case of $\alpha_{k}=1$ for all $k$ as

$$
\log \left(1+M^{2 q-1-\epsilon}\right) \lesssim \mathbb{E}[\log (1+Z)] \lesssim \log \left(1+M^{2 q-1+\epsilon}\right)
$$

for any sufficiently small $\epsilon>0$, where $Z=\max _{1 \leq k \leq K} Z_{k}$ and $x \lesssim y$ means $\lim _{M \rightarrow \infty} x / y \leq 1$.

Proof: The probability of the event $\left\{Z>M^{p}\right\}$ for any $p \in(-1,1)$ can be expressed as

$$
\begin{aligned}
\operatorname{Pr}\left\{\max _{k} Z_{k}>M^{p}\right\} & =1-\operatorname{Pr}\left\{Z_{k} \leq M^{p}\right\}^{K} \\
& =1-\left(1-\frac{1}{c M^{(1+p) / 2}}\right)^{K},
\end{aligned}
$$

where the second equality holds by Lemma 1 ( $c$ is a constant between $\frac{\pi}{4}$ and $\left.2 \pi\right)$. We now consider the second term in (22). Pick $p=2 q-1-\epsilon$ for small $\epsilon>0$ such that $p \in(-1,1)$. Then the second term is given by

$$
\begin{aligned}
\left(1-\frac{1}{c M^{(1+p) / 2}}\right)^{K} & =\left(1-\frac{1}{c M^{q-\frac{\epsilon}{2}}}\right)^{M^{q}} \\
& =e^{M^{q} \log \left(1-\frac{1}{c M^{q-\frac{\epsilon}{2}}}\right)} \\
& =e^{-\frac{1}{c} M^{\epsilon / 2}+O\left(\frac{1}{M^{2 q-\epsilon}}\right)} \\
& \rightarrow 0 \text { as } M \rightarrow \infty
\end{aligned}
$$

where we used the fact that $\log (1-x)=-x+O\left(x^{2}\right)$ for small $x$ in the third step. Therefore, in this case, we have $\operatorname{Pr}\left\{Z>M^{p}\right\} \rightarrow 1$ and thus $\mathbb{E}[\log (1+Z)]$ can be bounded as

$$
\begin{aligned}
\mathbb{E}[\log (1+Z)] & \geq \int_{M^{p}}^{M} \log (1+z) p(z) d z \\
& \geq \log \left(1+M^{p}\right) \int_{M^{p}}^{M} p(z) d z \\
& \rightarrow \log \left(1+M^{p}\right), \text { as } \quad M \rightarrow \infty .
\end{aligned}
$$


Hence, the claim on the lower bound follows. Now pick $p=$ $2 q-1+\epsilon$ for small $\epsilon>0$ such that $p \in(-1,1)$. Then, by the techiques used in (23)-(25), the second term in (22) can be computed as

$$
\begin{aligned}
\left(1-\frac{1}{c M^{(1+p) / 2}}\right)^{K} & =e^{-\frac{1}{c} M^{-\epsilon / 2}+O\left(\frac{1}{M^{2 q+\epsilon}}\right)} \\
& =1-O\left(\frac{1}{M^{\frac{\epsilon}{2}}}\right)
\end{aligned}
$$

where the second inequality holds by the identity $e^{x}=1+$ $O(x)$ for small $x$. Therefore, in this case, the probability of the event $\left\{Z>M^{p}\right\}$ is given by $O\left(\frac{1}{M^{\epsilon / 2}}\right)$. Using this, we have

$$
\begin{aligned}
\mathbb{E}[\log (1+Z)] & =\int_{M^{p}}^{M} \log (1+z) p(z) d z+\int_{0}^{M^{p}} \log (1+z) p(z) d z \\
& \leq \log (1+M) O\left(\frac{1}{M^{\epsilon / 2}}\right)+\log \left(1+M^{p}\right) \\
& \rightarrow \log \left(1+M^{p}\right), \text { as } M \rightarrow \infty .
\end{aligned}
$$

Hence, the claim on the upper bound follows.

Theorem 1 states that the RDB scheme under the assumption $\alpha_{k}=1, \forall k$ has asymptotically nontrivial performance, i.e., $\mathcal{R}_{1} \rightarrow \infty$, as $M \rightarrow \infty$, when $K=M^{q}$ with $q \in\left(\frac{1}{2}, 1\right)$. When $K=M^{q}$ with $q \in\left(0, \frac{1}{2}\right)$, on the other hand, the RDB scheme has trivial performance, i.e., $\mathcal{R}_{1} \rightarrow 0$, as $M \rightarrow \infty$. Therefore, $q=\frac{1}{2}$ is the transition point for the RDB scheme under the UR-LoS channel model, which determines the sufficiency or deficiency of MU gain. It is easy to see that

$$
\lim _{M \rightarrow \infty} \frac{\mathbb{E}[\log (1+Z)]}{\log (1+M)}=2 q-1,
$$

for $K=M^{q}$ with $q \in\left(\frac{1}{2}, 1\right)$, where $\log (1+M)$ is the rate of perfect beamforming based on perfect CSI at the transmitter. Thus, the RDB strategy under the assumption $\alpha_{k}=1, \forall k$ achieves $2 q-1$ fraction of the exact beamforming rate based on perfect CSI at the transmitter, when $K=M^{q}$ with $q \in\left(\frac{1}{2}, 1\right)$. Note that the maximum value of one for (27) is achieved if $q \uparrow 1$, i.e., the performance of perfect beamforming based on perfect CSI is achieved by the RDB scheme if the number of users increases linearly with the number $M$ of transmit antennas. It is also shown in [1] that the same is true for the UR-LOS channel with $\alpha_{k} \stackrel{i . i . d .}{\sim} \mathcal{C N}(0,1)$.

\section{Multiple Orthogonal Beams: Impact of TRAINING}

In this section, we consider what can be achieved by training for the RDB scheme. To train the network, we define $S$ random training beams equi-spaced in the normalized angle domain as

$$
\mathbf{w}_{b}=\mathbf{a}\left(\vartheta_{b}\right)=\mathbf{a}\left(\vartheta+\frac{2(b-1)}{S}\right), \text { for } b=1, \cdots, S,
$$

where $\vartheta \sim \operatorname{Unif}[-1,1]$, and the transmitter sequentially transmits the $S$ random beams to the downlink during the training period. We assume that the network is synchronized and hence each user knows the training beam index $b$ by looking at the corresponding training interval. Note that the difference between the normalized angles of two adjacent training beams is $\frac{2}{S}$ and the offset $\vartheta$ is randomly generated on
$[-1,1]$. (Note from (3) that $\mathbf{a}(\theta)$ is periodic in $\theta$ with period 2.) The equi-spaced beams are asymptotically orthogonal to one another, i.e.,

$$
\lim _{M \rightarrow \infty}\left|\mathbf{a}\left(\vartheta_{b_{1}}\right)^{H} \mathbf{a}\left(\vartheta_{b_{2}}\right)\right|=0 \text { for } b_{1} \neq b_{2},
$$

if $S=o(M)$. During the training period, each user computes the received power for each training beam and finds the maximum. In this way, each user knows its channel better and better as $S$ increases. When the training period is over, each user feeds back the maximum of its received power values for the $S$ training beams and the corresponding beam index. Then, the transmitter transmits a data stream to the user that has maximum received power with the corresponding beam $\mathbf{w}_{b}$. In this case, the corresponding rate $\mathcal{R}_{S}$ is given by

$$
\mathcal{R}_{S}=\mathbb{E}\left[\log \left(1+\max _{1 \leq k \leq K} \max _{1 \leq b \leq S}\left|\alpha_{k}\right|^{2} M\left|\mathbf{a}\left(\theta_{k}\right)^{H} \mathbf{a}\left(\vartheta_{b}\right)\right|^{2}\right)\right] \text {. }
$$

To compute the asymptotic value of $\mathcal{R}_{S}$, we first consider the case of $\left|\alpha_{k}\right|=1$ for all $k=1, \cdots, K$ as before. In this case, $\mathcal{R}_{S}$ can be lower and upper bounded asymptotically as follows:

Theorem 2: [1] For $K=M^{q}, S=M^{\ell}$ and any $\ell, q \in(0,1)$ such that $\ell+q<1$, we have asymptotic lower and upper bounds on $\mathcal{R}_{S}$ in the case of $\left|\alpha_{k}\right|=1, \forall k$ as

$$
\begin{aligned}
\log \left(1+M^{2(q+\ell)-1-\epsilon}\right) & \lesssim \mathbb{E}\left[\log \left(1+Z^{\prime}\right)\right] \\
& \lesssim \log \left(1+M^{2(q+\ell)-1+\epsilon}\right)
\end{aligned}
$$

for any sufficiently small $\epsilon>0$, where $Z^{\prime}=\max _{k} Z_{k}^{\prime}$ and $Z_{k}^{\prime}=\max _{b} M\left|\mathbf{a}\left(\theta_{k}\right)^{H} \mathbf{a}\left(\vartheta_{b}\right)\right|^{2}$.

Proof: Please see [1].

Theorem 2 states that the multi-beam training RDB scheme under the assumption $\alpha_{k}=1, \forall k$ has asymptotically nontrivial performance, i.e., $\mathcal{R}_{S} \rightarrow \infty$, as $M \rightarrow \infty$, when $K=M^{q}$ and $S=M^{\ell}$ with $\ell+q \in\left(\frac{1}{2}, 1\right)$. When $\ell+q \in\left(0, \frac{1}{2}\right)$, on the other hand, the multi-beam training RDB scheme has trivial performance, i.e., $\mathcal{R}_{S} \rightarrow 0$, as $M \rightarrow \infty$. Note that $q$ in Theorem 1 is replaced by $q+\ell$ in Theorem 2 , and the two theorems are the same except this. Now the role of training for RDB is clear from Theorem 2 . When the number of users in the network is not sufficient, i.e., $q<1 / 2$, training should be used to enhance the RDB performance so that $q+\ell>1 / 2$. The lack of MU gain can be compensated for by training. In the extreme case of $K=1$, i.e., $q=0, \ell$ should do all the work. Again, the performance transition point is $q+\ell=1 / 2$. Note that we have

$$
\lim _{M \rightarrow \infty} \frac{\mathbb{E}\left[\log \left(1+Z^{\prime}\right)\right]}{\log (1+M)}=2(q+\ell)-1 .
$$

for $K=M^{q}, S=M^{\ell}$ and any $\ell, q \in(0,1)$ such that $\frac{1}{2}<$ $\ell+q<1$, where $\log (1+M)$ is the rate of perfect beamforming based on perfect CSI. In the case of multi-beam training RDB, the maximum value of one for (32) can be achieved as the sum of MU gain factor $q$ and training factor $\ell$ approaches one.

Considering the randomness in the path gain, we can further show that for $\alpha_{k} \stackrel{i . i . d .}{\sim} \mathcal{C N}(0,1)$ for each $k$

$$
\lim _{M \rightarrow \infty} \frac{\mathcal{R}_{S}}{\mathbb{E}\left[\log \left(1+\max _{k}\left|\alpha_{k}\right|^{2} M\right)\right]}=2(q+\ell)-1,
$$

for $K=M^{q}, S=M^{\ell}$ and any $\ell, q \in(0,1)$ such that $\frac{1}{2}<$ $\ell+q<1[1]$. 


\section{NUMERICAL RESUlTS}

In this section, we provide some numerical results to validate our asymptotic analysis in the previous sections. We considered a mm-wave MU-MIMO downlink system with the UR-LoS channel model. All the expectations in the numerical results are average over 5000 channel realizations and we set $P_{t}=1$.

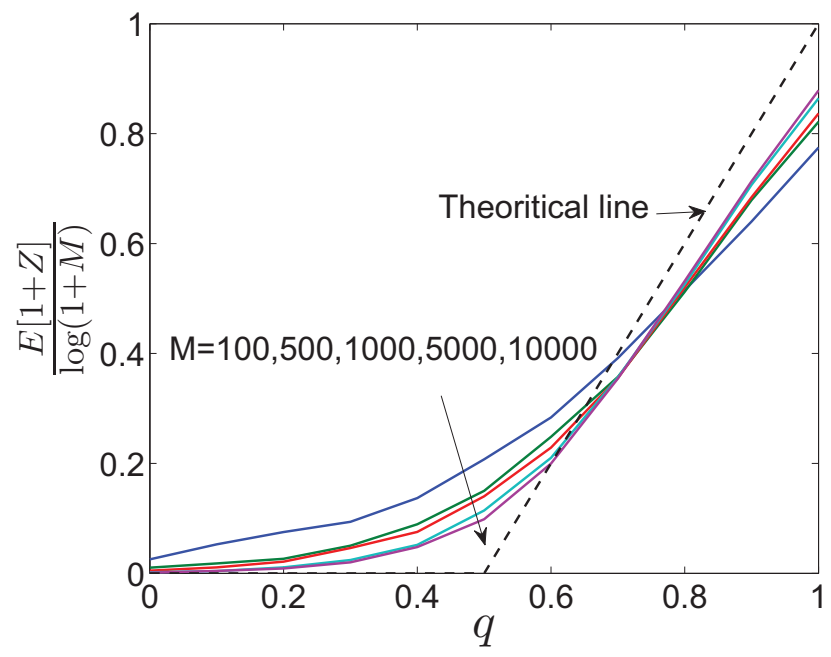

Fig. 1. The ratio of the RDB rate $\mathcal{R}_{1}$ to the rate with perfect CSI $\mathbb{E}[\log (1+$ $M)]$ versus $q$ for different $M[1]$

We first validate the result in Section IV. Fig. 1 shows the value of $\frac{\mathbb{E}[1+Z]}{\log (1+M)}$ versus $q$ for $M=$ $100,500,1000,5000,10000$. It is seen that the curve of $\frac{\mathbb{E}[1+Z]}{\log (1+M)}$ versus $q$ gradually converges to the theoretical line of $2 q-1$ for $q>\frac{1}{2}$ and 0 for $q \leq \frac{1}{2}$ as $M$ increases.

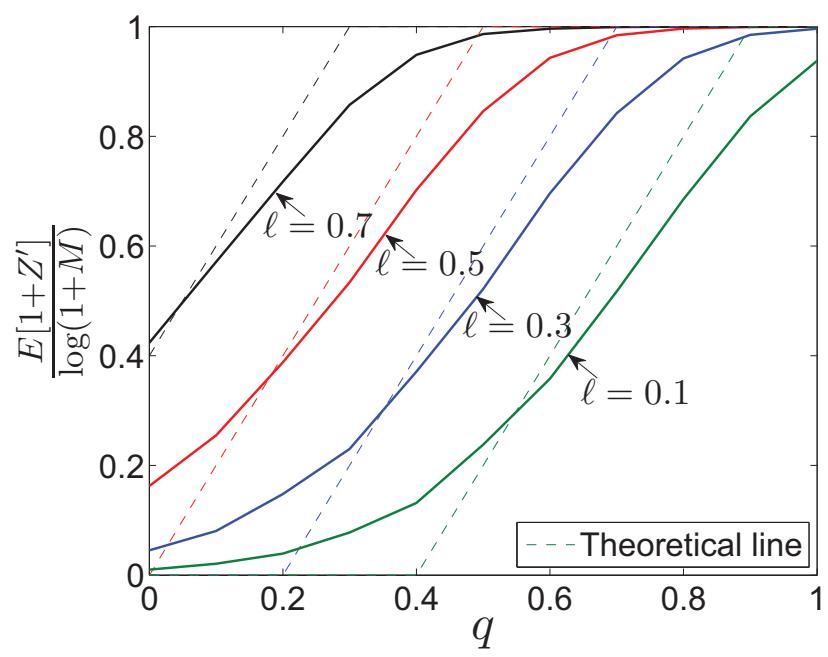

Fig. 2. The ratio of the RDB rate $\mathcal{R}_{S}$ to the rate with perfect $\mathrm{CSI} \mathbb{E}[\log (1+$ $M)$ ] versus $q$ for different $\ell$, when $M=1000$ [1]

Next, we considered the multi-beam training RDB scheme explained in Section V. Fig. 2 shows the ratio of the multibeam training RDB rate $\mathcal{R}_{S}$ to the rate with perfect CSI versus $q$ for different $\ell$, when $M=1000$. It is seen that the simulation curves roughly match the theoretical lines.

\section{CONCLUSION}

We have considered RDB with partial CSI to resolve the difficulties of channel estimation and CSI feedback in mmwave MU-MIMO downlink, and examined the associated MU gain and the impact of training, using asymptotic analysis, based on the UR-LoS channel model which is suitable for radio channels in the mm-wave band. We have shown that there exists a performance transition point in the number of users (relative to the number of antennas) for non-trivial performance of the RDB scheme and have revealed the interplay between the MU gain and training for RDB. When the number of users is less than the square root of the number of transmit antennas, training should be used to enhance the RDB performance. Furthermore, we have shown that as the product of the number of users and the number of equi-spaced training beams increases linearly with respect to the number of transmit antennas, the performance of perfect beamforming with perfect CSI can be achieved by RDB. The results here provide insights into system design in mm-wave MU-MIMO. The multi-beam multi-user selection case was studied in [1].

\section{REFERENCES}

[1] G. Lee, Y. Sung, and J. Seo, "Randomly-directional beamforming in millimeter-wave multi-user MISO downlink," submitted to IEEE Trans. Wireless Commun., May 2015.

[2] G. Lee, Y. Sung, and M. Kountouris, "On the performance of randomly directional beamforming between line-of-sight and rich scattering channels," in Proc. of IEEE SPAWC, Jun. 2015.

[3] M. Sharif and B. Hassibi, "On the capacity of MIMO broadcast channels with partial side information," IEEE Trans. Inf. Theory, vol. 51, pp. 506522, Feb. 2005.

[4] T. Yoo and A. Goldsmith, "On the optimality of multiantenna broadcast scheduling using zero-forcing beamforming," IEEE J. Sel. Areas Commun., vol. 24, pp. 528-541, Mar. 2006.

[5] A. Tomasoni and G. Caire and M. Ferrari and S. Bellini, "On the selection of semi-orthogonal users for zero-forcing beamforming," in Proc. IEEE ISIT, Jul. 2009.

[6] H. Hur, A. M. Tulino, and G. Caire, "Network MIMO with linear zeroforcing beamforming: Large system analysis, impact of channel estimation, and reduced-complexity scheduling," IEEE Trans. Inf. Theory, vol. 58, pp. 2911-2934, May 2012.

[7] J. Nam, A. Adhikary, J. Ahn, and G. Caire, "Joint spatial division and multiplexing: Opportunistic beamforming, user grouping and simplified downlink scheduling," IEEE J. Sel. Topics Signal Process., vol. 8, pp. $876-890$, Oct. 2014

[8] A. Alkhateeb, O. E. Ayach, G. Leus, and R. W. Heath Jr., "Channel estimation and hybrid precoding for millimeter wave cellular systems," IEEE J. Sel. Topics Signal Process., vol. 8, pp. 831 - 846, Oct. 2014.

[9] W. U. Bajwa, J. Haupt, A. M. Sayeed, and R. Nowak, "Compressed channel sensing: a new approach to estimating sparse multipath channels," Proc. IEEE, vol. 98, pp. 1058 - 1076, Jun. 2010.

[10] J. Seo, Y. Sung, G. Lee, and D. Kim, "Training beam sequence design for millimeter-wave MIMO systems: A POMDP framework," submitted to IEEE Trans. Wireless Commun., arXiv preprint arXiv:1410.3711, 2014.

[11] A. Sayeed and J. Brady, "Beamspace MIMO for high-dimensional multiuser communication at millimeter-wave frequencies," in Proc. IEEE Global Telecommun. Conf. (Globecom), pp. 3679 - 3684, Dec. 2013.

[12] H. Q. Ngo, E. G. larsson, and T. L Marzetta, "Aspects of favorable propagation in massive MIMO," in Proc. IEEE EUSIPCO 2014, pp. 76 - 80, Sep. 2014.

[13] T. S. Rappaport, E. Ben-Dor, J. N. Murdock, and Y. Qiao, "38 GHz and $60 \mathrm{GHz}$ angle-dependent propagation for cellular \& peer-to-peer wireless communications," in Proc. IEEE Int. Conf. Commun. (ICC), Jun. 2012.

[14] T. Bai, V. Desai, and R. W. Heath, "Millimeter wave cellular channel models for system evaluation," in Proc. IEEE ICNC 2014, pp. 178 182, Feb. 2014.

[15] R. S. Strichartz, The Way of Analysis. Sudbury, MA: Jones and Bartlett Publishers, 2000. 